\title{
Synthesis of Substituted Imidazoles via Organocatalysis
}

Doug E. Frantz, ${ }^{* a}$ Louis Morency, ${ }^{\dagger}$ Arash Soheili, ${ }^{\mathrm{b}}$ Jerry A. Murry, ${ }^{\mathrm{b}}$ Edward J. J. Grabowski, ${ }^{\mathrm{b}}$ and Richard D. Tillyer ${ }^{\mathrm{b}}$

${ }^{a}$ Department of Process Research, Merck Research Laboratories, Merck \& Co., 466 Devon Park Drive, Wayne, PA 19087

${ }^{b}$ Department of Process Research, Merck Research Laboratories, Merck \& Co., Rahway, NJ 07065

\section{Supporting Information}

General Methods: All reactions were performed under an atmosphere of nitrogen using dry solvents $(\mathrm{KF}<300 \mu \mathrm{g} / \mathrm{mL})$. The starting $\alpha$-amido sulfones were synthesized according to known procedures. ${ }^{1}{ }^{1} \mathrm{H}$ and ${ }^{13} \mathrm{C}$ NMR data for compounds 4-8 was obtained in $\mathrm{CD}_{3} \mathrm{CN}$ containing $1 \%$ trifluoroacetic acid (by volume) and in $\mathrm{CDCl}_{3}$ for compounds 10-18 using a Bruker Advance 300 spectrometer with chemical shifts referenced to the internal solvent signal. Chromatographic purification of products was accomplished using forced flow chromatography on silica gel (230 mesh) according to the method of Stille. ${ }^{2}$ Reactions were monitored by HPLC analysis on a HP 1100 system using a Waters Xterra $\mathrm{RP}_{18} 5 \mu \mathrm{m} 4.6 \mathrm{~mm}$ X 250mm column and acetonitrile / 20mM KH $\mathrm{KHO}_{4}$ as eluents. Enantiomeric excesses for compounds $\mathbf{1 1}$ and $\mathbf{1 2}$ were determined by HPLC analysis using a Chiracel OJ-H column and heptane / isopropanol as eluents. Melting points were determined on a PerkinElmer Pyris 1 differential scanning calorimeter and are uncorrected.

General Procedure for the Synthesis of Substituted Imidazoles: A 50mL flask was charged with the $\alpha$-amido suflone $(3.0 \mathrm{mmol}, 1.0 \mathrm{eq})$ and the thiazolium catalyst $(5-20$ $\mathrm{mol} \%)^{3}$ and purged with nitrogen for $15 \mathrm{~min}$. To the flask was added solvent ${ }^{4}(20 \mathrm{~mL})$ followed by the aldehyde $(3.3 \mathrm{mmol}, 1.1 \mathrm{eq})$ and the resulting mixture stirred and heated to $35^{\circ} \mathrm{C}$. Triethylamine ( $45 \mathrm{mmol}, 15 \mathrm{eq}$ ) was added in one portion via syringe and the corresponding reaction was monitored by HPLC analysis for consumption of the amido sulfone. After the reaction was complete, the solvent was removed by simple concentration followed by the addition of $\mathrm{EtOH}(20 \mathrm{~mL})$, acetic acid (15 mmol, 5 equiv) and the appropriate amine ( $15 \mathrm{mmol}, 5$ equiv) in that order to the same reaction flask. If the resulting amine salt precipitates from the reaction mixture, then enough ethanol was added to obtain a homogeneous reaction mixture. The reaction was then heated to reflux and monitored by HPLC for consumption of the keto-amide. Once the reaction is complete (typical reaction times $\sim 12-24 \mathrm{~h}$ ), the corresponding substituted imidazole was isolated by either an aqueous workup followed by flash chromatography or by direct crystallization from the reaction mixture by the addition of water.

\footnotetext{
${ }^{1}$ Murry, J. A.; Frantz, D. E.; Soheili, A.; Tillyer, R.; Grabowski, E. J. J.; Reider, P. J. J. Am. Chem. Soc. 2001, 123, 9696-9697.

${ }^{2}$ Still, W. C., Ammon, H. L., DeShong, P.J. Am. Chem. Soc. 1995, 117, 5166.

${ }^{3}$ Aliphatic and electron-rich aromatic aldehydes typically require higher levels of thiazolium catalyst (15$20 \mathrm{~mol} \%$ ) due to lower reactivity.

${ }^{4}$ Both $\mathrm{CH}_{2} \mathrm{Cl}_{2}$ and THF were found to be suitable solvents with the former being preferred in most cases.
} 


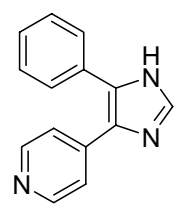

4-(5-phenyl-1H-imidazole-4-yl)pyridine (4): Isolated in 76\% yield by silica gel chromatography as a white crystalline solid, m.p. $=228.6{ }^{\circ} \mathrm{C}$. ${ }^{1} \mathrm{H}$ NMR (300.13 MHz, 1\% TFA in $\mathrm{CD}_{3} \mathrm{CN}$ ) $\delta 13.0$ (br s, $\left.1 \mathrm{H}\right), 8.57$ (d, $2 \mathrm{H}, \mathrm{J}=7.1 \mathrm{~Hz}$ ), $8.55(\mathrm{~s}, 1 \mathrm{H}), 7.99(\mathrm{~d}, 2 \mathrm{H}, \mathrm{J}=7.1 \mathrm{~Hz}), 7.62-7.49(\mathrm{~m}, 5 \mathrm{H}) ;{ }^{13} \mathrm{C}$ NMR $(75.48$ $\mathrm{MHz}, 1 \% \mathrm{TFA}$ in $\left.\mathrm{CD}_{3} \mathrm{CN}\right) \delta 149.0,142.3,137.1,134.8,129.8,129.7,129.3$, $128.9,122.2$.

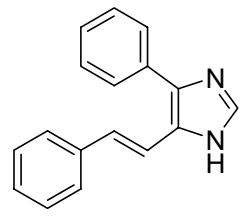

4-phenyl-5-[(E)-2-phenylvinyl]-1H-imidazole (5): Isolated by direct crystallization from the reaction mixture in $47 \%$ yield and by flash chromatography in $68 \%$ yield as a light yellow crystalline solid, m.p. $=$ 182.5. ${ }^{1} \mathrm{H}$ NMR (300.13 MHz, $1 \%$ TFA in $\mathrm{CD}_{3} \mathrm{CN}$ ) $\delta 12.3$ (br s, $1 \mathrm{H}$ ), $8.63(\mathrm{~s}, 1 \mathrm{H}), 7.61-7.51(\mathrm{~m}, 7 \mathrm{H}), 7.42-7.30(\mathrm{~m}, 3 \mathrm{H}), 7.25(\mathrm{~d}, 1 \mathrm{H}, \mathrm{J}=16.7$ $\mathrm{Hz}), 7.07(\mathrm{~d}, 1 \mathrm{H}, \mathrm{J}=16.7 \mathrm{~Hz}) ;{ }^{13} \mathrm{C}$ NMR $\left(75.48 \mathrm{MHz}, 1 \%\right.$ TFA in $\left.\mathrm{CD}_{3} \mathrm{CN}\right) \delta 135.9$, $134.4,132.7,130.0,129.4,129.2,128,8,128.7,128.3,127.5,127.3,126.7,112.7$.

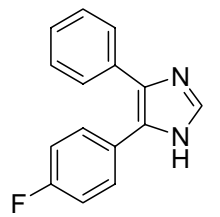

5-(4-fluorophenyl)-4-phenyl-1H-imidazole (6): Isolated by flash chromatography in $82 \%$ yield as a white crystalline solid, m.p. $=215.7^{\circ} \mathrm{C}$. ${ }^{1} \mathrm{H}$ NMR $\left(300.13 \mathrm{MHz}, 1 \%\right.$ TFA in $\left.\mathrm{CD}_{3} \mathrm{CN}\right) \delta 12.5(\mathrm{br} \mathrm{s}, 1 \mathrm{H}), 8.65(\mathrm{~s}, 1 \mathrm{H})$, $7.50-7.38(\mathrm{~m}, 7 \mathrm{H}), 7.22-7.13(\mathrm{tt}, 2 \mathrm{H}, \mathrm{J}=8.9 \mathrm{~Hz}, 2.2 \mathrm{~Hz}) ;{ }^{13} \mathrm{C}$ NMR $(75.48$ $\mathrm{MHz}, 1 \%$ TFA in $\left.\mathrm{CD}_{3} \mathrm{CN}\right) \delta 166.1,162.9,135.1,132.1,132.0,130.8$, $130.4,130.2,129.6,129.3,128.7,117.5,117.2$.

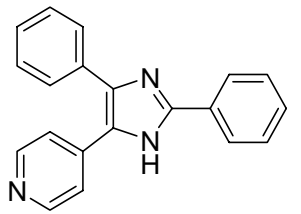

4-(2,4-diphenyl-1H-imidazole-5-yl)pyridine (7): Isolated by flash chromatography in $78 \%$ yield as an off-white crystalline solid, m.p. $=$ $289.5^{\circ} \mathrm{C}$. ${ }^{1} \mathrm{H}$ NMR $\left(300.13 \mathrm{MHz}, 1 \%\right.$ TFA in $\left.\mathrm{CD}_{3} \mathrm{CN}\right) \delta 13.2(\mathrm{br} \mathrm{s}$, $1 \mathrm{H}), 8.51(\mathrm{~d}, 2 \mathrm{H}, \mathrm{J}=7.0 \mathrm{~Hz}), 8.07(\mathrm{~d}, 2 \mathrm{H}, \mathrm{J}=7.0 \mathrm{~Hz}), 8.06-8.01(\mathrm{~m}, 2$ $\mathrm{H}), 7.62-7.51(\mathrm{~m}, 8 \mathrm{H}) ;{ }^{13} \mathrm{C}$ NMR $\left(75.48 \mathrm{MHz}, 1 \% \mathrm{TFA}\right.$ in $\left.\mathrm{CD}_{3} \mathrm{CN}\right) \delta$ $149.5,149.1,143.0,138.1,133.0,132.0,130.9,130.8,130.7,129.2,128.8,128.3,127.0$, 125.1 .

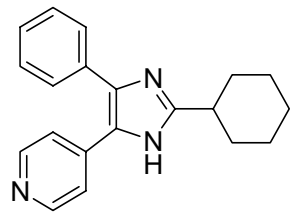

4-(2-cyclohexyl-4-phenyl-1H-imidazol-5-yl)pyridine (8): Isolated by direct crystallization from the reaction mixture in $55 \%$ yield and by flash chromatography in $82 \%$ yield as a white crystalline solid, m.p. $=$ $254.5^{\circ} \mathrm{C} .{ }^{1} \mathrm{H}$ NMR $\left(300.13 \mathrm{MHz}, 1 \% \mathrm{TFA}\right.$ in $\mathrm{CD}_{3} \mathrm{CN}$ ) $\delta 11.1$ (br s, $1 \mathrm{H}), 8.62(\mathrm{~d}, 2 \mathrm{H}, \mathrm{J}=7.0 \mathrm{~Hz}), 7.95(\mathrm{~d}, 2 \mathrm{H}, \mathrm{J}=7.0 \mathrm{~Hz}), 7.65-7.49(\mathrm{~m}$, $5 \mathrm{H}), 3.21-3.09$ (tt, $1 \mathrm{H}, \mathrm{J}=12.1 \mathrm{~Hz}, 3.4 \mathrm{~Hz}$ ), 2.15-2.06 (br d, $2 \mathrm{H}, \mathrm{J}=12.9 \mathrm{~Hz}), 1.94-1.84$ (m, 2H), 1.80-1.60 (m, 3H), 1.52-1.24 (m, 3H); ${ }^{13} \mathrm{C}$ NMR (75.48 MHz, 1\% TFA in $\left.\mathrm{CD}_{3} \mathrm{CN}\right) \delta 154.5,145.1,142.9,134.1,130.4,129.4,129.1,127.1,125.1,123.5,36.4$, $30.6,25.4,25.1$.

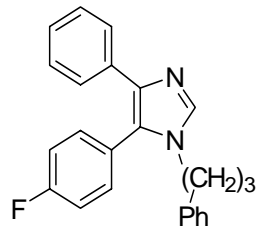

5-(4-fluorophenyl)-4-phenyl-1-(3-phenylpropyl)-1H-imidazole

(9): Isolated by direct crystallization from the reaction mixture in 35\% yield and by flash chromatography in $58 \%$ yield as a light yellow crystalline solid, m.p. $=118.2 .{ }^{1} \mathrm{H}$ NMR $\left(300.13 \mathrm{MHz}, \mathrm{CDCl}_{3}\right) \delta 7.62(\mathrm{~s}, 1 \mathrm{H}), 7.48-$ $7.42(\mathrm{~m}, 2 \mathrm{H}), 7.31-7.10(\mathrm{~m}, 10 \mathrm{H}), 7.06-7.01(\mathrm{~m}, 2 \mathrm{H}), 3.80(\mathrm{t}, 2 \mathrm{H}, \mathrm{J}=$ 7.3Hz), $2.54(\mathrm{t}, 2 \mathrm{H}, 7.4 \mathrm{~Hz}), 1.91(\mathrm{p}, 2 \mathrm{H}, \mathrm{J}=7.6 \mathrm{~Hz}) ;{ }^{13} \mathrm{C} \mathrm{NMR}\left(75.48 \mathrm{MHz}, \mathrm{CDCl}_{3}\right) \delta$ 
$164.6,161.3,140.2,138.5,136.9,134.4,132.7,132.6,128.6,128.3,127.3,126.8,126.6$, $126.5,126.3,116.5,116.2,44.5,32.5,32.0$.

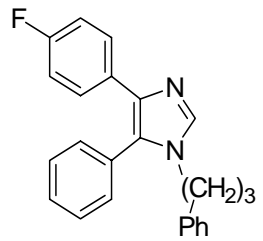

4-(4-fluorophenyl)-5-phenyl-1-(3-phenylpropyl)-1H-imidazole $\quad(10)$ : Isolated by direct crystallization from the reaction mixture in $42 \%$ yield and by flash chromatography in $61 \%$ yield as a white crystalline solid, m.p. $=116.4{ }^{\circ} \mathrm{C}$. ${ }^{1} \mathrm{H}$ NMR $\left(300.13 \mathrm{MHz}, \mathrm{CDCl}_{3}\right) \delta 7.59(\mathrm{~s}, 1 \mathrm{H}), 7.48-$ $7.40(\mathrm{~m}, 5 \mathrm{H}), 7.34-7.15(\mathrm{~m}, 5 \mathrm{H}), 7.07-7.01(\mathrm{~m}, 2 \mathrm{H}), 6.89(\mathrm{tt}, 2 \mathrm{H}, \mathrm{J}=8.9$ $\mathrm{Hz}, 2.1 \mathrm{~Hz}), 3.82$ (t, 2H, J = 7.3 Hz), 2.53, (t, 2H, J = 7.5 Hz), 1.90 (p, 2H, J = 7.7 Hz); ${ }^{13} \mathrm{C}$ NMR $\left(75.48 \mathrm{MHz}, \mathrm{CDCl}_{3}\right) \delta 163.3,160.0,140.3,137.5,136.8,130.8,130.7,129.2$, $128.8,128.6,128.2,128.1,126.3,115.2,114.9,44.7,32.5,32.0$.

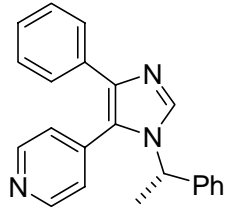

4-\{4-phenyl-1-[(1S)-1-phenylethyl]-1H-imidazol-5-yl\}pyridine

(11): Isolated by flash chromatography in $83 \%$ yield and $>98 \%$ ee as a white crystalline solid, m.p. $=157.5^{\circ} \mathrm{C} .{ }^{1} \mathrm{H}$ NMR $\left(300.13 \mathrm{MHz}, \mathrm{CDCl}_{3}\right) \delta 8.59$ $(\mathrm{d}, 2 \mathrm{H}, \mathrm{J}=6.0 \mathrm{~Hz}), 7.86(\mathrm{~s}, 1 \mathrm{H}), 7.43(\mathrm{~m}, 2 \mathrm{H}), 7.36-7.16(\mathrm{~m}, 7 \mathrm{H}), 7.04(\mathrm{~d}$, $2 \mathrm{H}, \mathrm{J}=6.0 \mathrm{~Hz}), 6.99-6.94(\mathrm{~m}, 2 \mathrm{H}), 5.15(\mathrm{q}, 1 \mathrm{H}, \mathrm{J}=7.1 \mathrm{~Hz}), 1.86(\mathrm{~d}, 3 \mathrm{H}, \mathrm{J}$ $=7.1 \mathrm{~Hz}) ;{ }^{13} \mathrm{C}$ NMR $\left(75.48 \mathrm{MHz}, \mathrm{CDCl}_{3}\right) \delta 150.4,141.6,139.7,139.4,135.6,133.8$, 129.0, 128.4, 128.1 127.0, 126.0, 125.6, 55.2, 22.7. Enantiomeric excess determined by HPLC analysis using a Chiracel OJ-H column and heptane / isopropanol (85:15) as eluents (flow rate $=1.0 \mathrm{~mL} / \mathrm{min}, 210 \mathrm{~nm}$ ). Retention time of $R$-isomer $=6.08 \mathrm{~min}, S$ isomer $=8.22 \mathrm{~min})$.

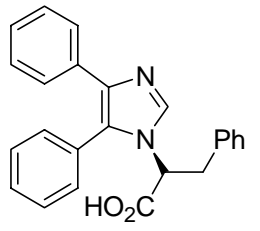

(2S)-2-(4,5-diphenyl-1H-imidazol-1-yl)-3-phenylpropanoic acid (12): Isolated by direct crystallization in $48 \%$ yield and by flash chromatography in $73 \%$ yield both in $>98 \%$ ee as a viscous oil. ${ }^{1} \mathrm{H}$ NMR $\left(300.13 \mathrm{MHz}, \mathrm{CD}_{3} \mathrm{OD}\right) \delta 8.21(\mathrm{~s}, 1 \mathrm{H}), 7.38-7.02(\mathrm{~m}, 11 \mathrm{H}), 6.93-6.90(\mathrm{~m}$, $2 \mathrm{H}), 6.75(\mathrm{~d}, 2 \mathrm{H}, \mathrm{J}=7.0 \mathrm{~Hz}), 4.49(\mathrm{dd}, 1 \mathrm{H}, \mathrm{J}=11.3 \mathrm{~Hz}, 3.9 \mathrm{~Hz}), 3.47$ $(\mathrm{dd}, 1 \mathrm{H}, \mathrm{J}=14.1 \mathrm{~Hz}, 3.9 \mathrm{~Hz}), 3.29-3.18(\mathrm{~m}, 1 \mathrm{H}) ;{ }^{13} \mathrm{C} \mathrm{NMR}\left(75.48 \mathrm{MHz}, \mathrm{CD}_{3} \mathrm{OD}\right) \delta$ $174.5,137.7,136.4,135.1$, 133.6, 131.0, 129.7, 129.6, 128.7, 128.6, 128.5, 128.2 , 127.7, $126.5,126.4,126.2,62.4,40.0$. Enantiomeric excess determined by HPLC analysis using a Chiracel AD column with isopropanol/heptane/TFA $(25: 75: 0.1)$ as eluents (flow rate = $1.0 \mathrm{~mL} / \mathrm{min}, 210 \mathrm{~nm}$ ). Retention time of $S$-isomer at $6.2 \mathrm{~min}, R$-isomer at $8.4 \mathrm{~min}$.

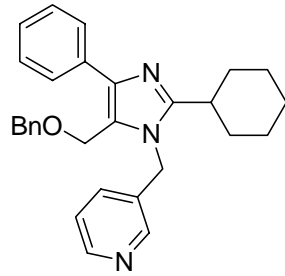

3-(\{5-[(benzyloxy)methyl]-2-cyclohexyl-4-phenyl-1H-imidazol-1yl\}methyl)pyridine (13): Isolated by flash chromatography in $22 \%$ yield as a white crystalline solid, m.p. $=90.4{ }^{\circ} \mathrm{C} .{ }^{1} \mathrm{H}$ NMR $(300.13$ $\left.\mathrm{MHz}, \mathrm{CDCl}_{3}\right) \delta 8.53(\mathrm{dd}, 1 \mathrm{H}, \mathrm{J}=4.3 \mathrm{~Hz}, 2.2 \mathrm{~Hz}), 8.36(\mathrm{~s}, 1 \mathrm{H}), 7.61$ (m, 2H), 7.40-7.17 (m, 11H), $5.18(\mathrm{~s}, 2 \mathrm{H}), 4.47(\mathrm{~s}, 2 \mathrm{H}), 4.41(\mathrm{~s}, 2 \mathrm{H})$, $2.53(\mathrm{~m}, 1 \mathrm{H}), 1.87-1.64(\mathrm{~m}, 7 \mathrm{H}), 1.38-1.15(\mathrm{~m}, 3 \mathrm{H}) ;{ }^{13} \mathrm{C}$ NMR $(75.48$ $\left.\mathrm{MHz}, \mathrm{CDCl}_{3}\right) \delta 153.4,149.1,147.7,141.1,137.5,134.7,133.7,133.0,128.5,128.4$, $128.3,128.0,127.0,123.8,122.9,72.3,61.1,44.5,36.4,32.1,26.4,25.7$.

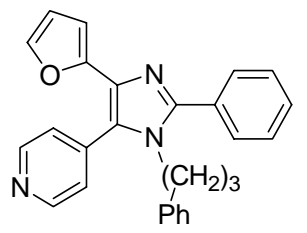

4-[4-(2-furyl)-2-phenyl-1-(3-phenylpropyl)-1H-imidazol-5yl]pyridine (14): Isolated by flash chromatography in $76 \%$ yield as a 
light orange solid, m.p. $=114.7^{\circ} \mathrm{C} .{ }^{1} \mathrm{H}$ NMR $\left(300.13 \mathrm{MHz}, \mathrm{CDCl}_{3}\right) \delta 8.70(\mathrm{dd}, 2 \mathrm{H}, \mathrm{J}=$ $6.0 \mathrm{~Hz}, 2.8 \mathrm{~Hz}), 7.65-7.58(\mathrm{~m}, 2 \mathrm{H}), 7.50-7.44(\mathrm{~m}, 3 \mathrm{H}), 7.35$ (dd, 2H, J = 6.0 Hz, $2.8 \mathrm{~Hz})$, 7.20-7.15 (m, 3H), 6.84-6.78 (m, 2H), $6.44(\mathrm{dd}, 1 \mathrm{H}, \mathrm{J}=3.6 \mathrm{~Hz}, 0.7 \mathrm{~Hz}), 6.35(\mathrm{dd}, 1 \mathrm{H}, \mathrm{J}=$ $3.4 \mathrm{~Hz}, 1.8 \mathrm{~Hz}), 3.95$ (t, 2H, J = $7.8 \mathrm{~Hz}), 2.29(\mathrm{t}, 2 \mathrm{H}, \mathrm{J}=7.3 \mathrm{~Hz}), 1.64(\mathrm{p}, 2 \mathrm{H}, \mathrm{J}=7.5$ $\mathrm{Hz}) ;{ }^{13} \mathrm{C}$ NMR $\left(75.48 \mathrm{MHz}, \mathrm{CDCl}_{3}\right) \delta 150.2,149.6,149.2,141.5,139.8,138.7,131.9$, $130.5,129.4,129.2,128.8,128.5,128.0,126.2,125.2,111.1,106.2,44.5,32.3,31.5$.

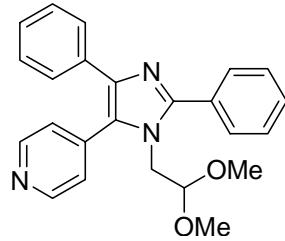

4-[1-(2,2-dimethoxyethyl)-2,4-diphenyl-1H-imidazol-5-yl]pyridine (15): Isolated by flash chromatography in $80 \%$ as a white crystalline solid, m.p. $=175.4{ }^{\circ} \mathrm{C} .{ }^{1} \mathrm{H}$ NMR $\left(300.13 \mathrm{MHz}, \mathrm{CDCl}_{3}\right) \delta 8.72(\mathrm{br} \mathrm{d}$, $2 \mathrm{H}, \mathrm{J}=5.9 \mathrm{~Hz}), 7.77-7.71(\mathrm{~m}, 2 \mathrm{H}), 7.55-7.46(\mathrm{~m}, 5 \mathrm{H}), 7.40(\mathrm{~d}, 2 \mathrm{H}, \mathrm{J}=$ $5.9 \mathrm{~Hz}), 7.28-7.20(\mathrm{~m}, 3 \mathrm{H}), 4.11(\mathrm{~d}, 2 \mathrm{H}, \mathrm{J}=5.5 \mathrm{~Hz}), 3.96(\mathrm{t}, 1 \mathrm{H}, \mathrm{J}=$ $5.3 \mathrm{~Hz}), 3.05(\mathrm{~s}, 6 \mathrm{H}) ;{ }^{13} \mathrm{C} \mathrm{NMR}\left(75.48 \mathrm{MHz}, \mathrm{CDCl}_{3}\right) \delta 150.6,149.6,139.7,139.4,133.8$, $130.9,129.5,129.3,128.8,128.3,127.5,127.2,127.1,125.7,103.0,55.0,46.9$.

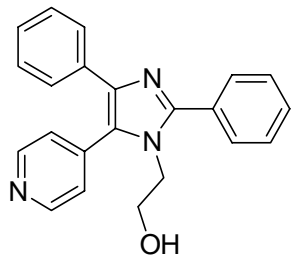

2-(2,4-diphenyl-5-pyridine-4-yl-1H-imidazol-1-yl)ethanol

(16):

Isolated by flash chromatography in $75 \%$ yield as a white crystalline solid, m.p. $=186.7{ }^{\circ} \mathrm{C} .{ }^{1} \mathrm{H}$ NMR $\left(300.13 \mathrm{MHz}, \mathrm{CDCl}_{3}\right) \delta 8.55$ (dd, $2 \mathrm{H}, \mathrm{J}=6.1 \mathrm{~Hz}, 2.9 \mathrm{~Hz}), 7.62-7.57(\mathrm{~m}, 2 \mathrm{H}), 7.48-7.39(\mathrm{~m}, 5 \mathrm{H}), 7.33$ $(\mathrm{dd}, 2 \mathrm{H}, \mathrm{J}=6.1 \mathrm{~Hz}, 2.9 \mathrm{~Hz}), 7.26-7.20(\mathrm{~m}, 3 \mathrm{H}), 4.51-4.20$ (br s, $1 \mathrm{H})$, $4.02(\mathrm{t}, 2 \mathrm{H}, \mathrm{J}=6.0 \mathrm{~Hz}), 3.41(\mathrm{t}, 2 \mathrm{H}, \mathrm{J}=6.0 \mathrm{~Hz}) ;{ }^{13} \mathrm{C} \mathrm{NMR}(75.48$ $\left.\mathrm{MHz} \mathrm{CDCl}_{3}\right) \delta 150.1,149.2,139.9,139.4,133.5,130.5,129.4,129.3,128.8,128.4$, $127.6,127.2,126.9,125.8,60.8,45.8$.

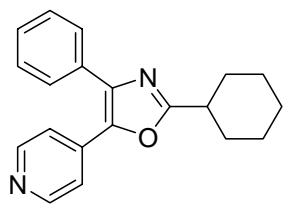

4-(2-cyclohexyl-4-phenyl-1,3-oxazol-5-yl)pyridine (17): A 50mL flask was charged with the $\alpha$-amido sulfone $(3.0 \mathrm{mmol}, 1.0 \mathrm{eq})$ and the thiazolium catalyst $(0.15 \mathrm{mmol}, 5 \mathrm{~mol} \%)$ and purged with nitrogen for $15 \mathrm{~min}$. To the flask was added $\mathrm{CH}_{2} \mathrm{Cl}_{2}(20 \mathrm{~mL})$ followed by $4-$ pyridinecarboxaldehyde $(3.3 \mathrm{mmol}, 1.1 \mathrm{eq})$ and the resulting mixture stirred and heated to $35{ }^{\circ} \mathrm{C}$. Triethylamine $(45 \mathrm{mmol}, 15 \mathrm{eq})$ was added in one portion via syringe and the corresponding reaction was monitored by HPLC analysis for consumption of the tosyl-amide. After the reaction was complete, it was cooled to $25{ }^{\circ} \mathrm{C}$ followed by the addition of $\mathrm{I}_{2}\left(6.0 \mathrm{mmol}, 2.0\right.$ equiv) and $\mathrm{PPh}_{3}(6.0 \mathrm{mmol}, 2.0$ equiv $) .^{5}$ The resulting reaction was allowed to proceed overnight at ambient temperatutre. The reaction was quenched with aqueous $\mathrm{Na}_{2} \mathrm{~S}_{2} \mathrm{O}_{3}$ and the resulting organic layer dried over $\mathrm{Na}_{2} \mathrm{SO}_{4}$. The oxazole was isolated by flash chromatography in $77 \%$ yield as a white crystalline solid, m.p. $=82.7{ }^{\circ} \mathrm{C} .{ }^{1} \mathrm{H}$ NMR $\left(300.13 \mathrm{MHz}, \mathrm{CDCl}_{3}\right) \delta 8.56(\mathrm{dd}, 2 \mathrm{H}, \mathrm{J}=4.5$ $\mathrm{Hz}, 1.6 \mathrm{~Hz}), 7.65-7.61(\mathrm{~m}, 2 \mathrm{H}), 7.46(\mathrm{dd}, 2 \mathrm{H}, \mathrm{J}=4.5 \mathrm{~Hz}, 1.6 \mathrm{~Hz}), 7.45-7.40(\mathrm{~m}, 3 \mathrm{H})$, $2.91(\mathrm{tt}, 1 \mathrm{H}, \mathrm{J}=11.4 \mathrm{~Hz}, 3.6 \mathrm{~Hz}), 2.21-2.12(\mathrm{~m}, 2 \mathrm{H}), 1.95-1.83(\mathrm{~m}, 2 \mathrm{H}), 1.80-1.62(\mathrm{~m}$, $3 \mathrm{H}), 1.50-1.26(\mathrm{~m}, 3 \mathrm{H}) ;{ }^{13} \mathrm{C} \mathrm{NMR}\left(75.48 \mathrm{MHz}, \mathrm{CDCl}_{3}\right) \delta 168.3,150.2,142.0,138.9$, $136.4,132.1,128.9,128.8,128.4,119.4,37.7,30.7,25.8,25.7$.

\footnotetext{
${ }^{5}$ Wipf, P.; Miller, C. P. J. Org. Chem. 1993, 58, 3604-3606.
} 


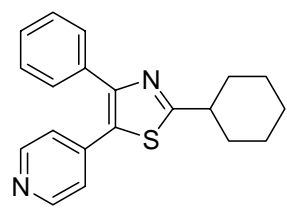

4-(2-cyclohexyl-4-phenyl-1,3-thiazol-5-yl)pyridine (18): A $50 \mathrm{~mL}$ flask was charged with the $\alpha$-amido sulfone $(3.0 \mathrm{mmol}, 1.0 \mathrm{eq})$ and the thiazolium catalyst $(0.15 \mathrm{mmol}, 5 \mathrm{~mol} \%)$ and purged with nitrogen for $15 \mathrm{~min}$. To the flask was added $\mathrm{CH}_{2} \mathrm{Cl}_{2}(20 \mathrm{~mL})$ followed by $4-$ pyridinecarboxaldehyde $(3.3 \mathrm{mmol}, 1.1 \mathrm{eq})$ and the resulting mixture stirred and heated to $35^{\circ} \mathrm{C}$. Triethylamine ( $\left.45 \mathrm{mmol}, 15 \mathrm{eq}\right)$ was added in one portion via syringe and the corresponding reaction was monitored by HPLC analysis for consumption of the tosyl-amide. After the reaction was complete, the solvent was removed and the crude mixture dissolved in fresh toluene $(20 \mathrm{~mL})$. Lawesson's reagent (4.5 mmol, 1.5 equiv) was added and the resulting reaction was allowed to reflux overnight. The reaction was cooled to ambient temperature followed by an aqueous workup. The thiazole was isolated by flash chromatography in $50 \%$ yield as a white crystalline solids, m.p. $=146.8{ }^{\circ} \mathrm{C} .{ }^{1} \mathrm{H}$ NMR $\left(300.13 \mathrm{MHz}, \mathrm{CDCl}_{3}\right) \delta 8.51(\mathrm{dd}, 2 \mathrm{H}, \mathrm{J}=$ 4.6 Hz, 1.5 Hz), 7.51-7.48 (m, 2H), 7.35-7.32 (m, 3H), 7.22 (dd, 2H, J = 4.6 Hz, 1.5 Hz), 3.12-3.02 (tt, $1 \mathrm{H}, \mathrm{J}=11.4 \mathrm{~Hz}, 3.5 \mathrm{~Hz}), 2.28-2.18(\mathrm{~m}, 2 \mathrm{H}), 1.93-1.72(\mathrm{~m}, 3 \mathrm{H}), 1.68-1.27$ $(\mathrm{m}, 5 \mathrm{H}) ;{ }^{13} \mathrm{C}$ NMR $\left(75.48 \mathrm{MHz}, \mathrm{CDCl}_{3}\right) \delta 176.6,151.4,150.1,140.7,134.6,129.2$, $128.6,128.5,128.4,123.7,43.0,33.8,26.1,25.8$. 